\title{
Let-7a inhibits proliferation and promotes apoptosis of human asthmatic airway smooth muscle cells
}

\author{
YAN CHEN $^{1}$, LUJUN QIAO $^{1}$, ZEWEN ZHANG $^{2}$, GUOXIN HU $^{1}$, JIAN ZHANG $^{1}$ and HONGJIA LI ${ }^{2}$ \\ ${ }^{1}$ Department of Critical Care Medicine, Shengli Oilfield Center Hospital, Dongying, Shandong 257000; \\ ${ }^{2}$ Department of Respiratory Disease, Qianfoshan Hospital, Shandong University, Jinan, Shandong 250014, P.R. China
}

Received June 30, 2018; Accepted December 13, 2018

DOI: $10.3892 /$ etm.2019.7363

\begin{abstract}
The present study aimed to examine the changes of let-7a expression in asthmatic airway smooth muscle cells (ASMCs) and to analyze its effect on the proliferation and apoptosis of ASMCs, as well as the potential mechanism of action. Let-7a expression levels in ASMCs from asthmatic and non-asthmatic subjects were detected using reverse transcription-quantitative polymerase chain reaction (RT-qPCR) analysis. Furthermore, let-7a mimics were transfected in vitro into ASMCs isolated from asthmatic patients, and the effect of let-7a on ASMC proliferation was examined using a Cell Counting Kit-8. In addition, the influence of let-7a on ASMC apoptosis was detected using flow cytometry and a caspase-3/7 activity assay. Target genes of let-7a were predicted using bioinformatics software, and the direct regulatory effect of let-7a on the potential target gene signal transducer and activator of transcription 3 (STAT3) was verified through a dual-luciferase reporter gene assay combined with RT-qPCR and western blot analysis. The results demonstrated that let-7a expression was significantly lower in ASMCs of asthmatic subjects compared with that in ASMCs of normal subjects. Furthermore, upregulation of let-7a expression in asthmatic ASMCs markedly inhibited cell proliferation and promoted cell apoptosis. The results of the dual-luciferase reporter gene assay indicated that let-7a selectively binds with the 3'-untranslated region of the STAT3 mRNA. In addition, let-7a mimics evidently reduced the mRNA and protein expression levels of STAT3 in asthmatic ASMCs. In conclusion, the present study demonstrates that let-7a expression is downregulated in ASMCs from asthmatic patients. Furthermore, let-7a suppresses the proliferation and promotes apoptosis of human asthmatic ASMCs, which may, at least partially, be associated with the downregulation of STAT3 expression.
\end{abstract}

Correspondence to: Dr Hongjia Li, Department of Respiratory Disease, Qianfoshan Hospital, Shandong University, 16766 Jingshi Road, Jinan, Shandong 250014, P.R. China

E-mail: lihongjiastar@yeah.net

Key words: asthma, airway smooth muscle cells, airway remodeling, let-7a, signal transducer and activator of transcription 3

\section{Introduction}

Bronchial asthma is a chronic airway inflammatory disease characterized by airway hyperreactivity and reversible flow limitation (1). Epidemiological investigations indicate that $\sim 0.3$ billion patients currently suffer from asthma and that 25,000 asthma-associated deaths occur per annum worldwide. Furthermore, the morbidity and mortality of asthma exhibit increasing trends, rendering asthma a major global public health problem (2). Chronic airway inflammation-induced airway remodeling is an important step in the pathogenesis of asthma (3). Airway remodeling mainly involves chronic inflammation-induced massive proliferation of airway smooth muscle cells (ASMCs) and extracellular matrix (ECM) deposition. The proliferation and hypertrophy of ASMCs have a particularly important role in airway remodeling, and are considered as important features thereof (4). In addition, the extent of ASMC proliferation is positively correlated with the severity of asthma (5).

Various factors, including T-helper cell type $1 / 2$ cytokine imbalances, cytokines, matrix metallopeptidase and tissue inhibitor of metallopeptidases, genetic factors, neuromodulation and gene mutation, have important roles in the occurrence and development of asthma (6,7). MicroRNAs (miRNAs/miRs) are a class of highly evolutionarily conserved, small-molecular, non-coding RNAs with a length of 21-23 nt. They interact with the mRNAs of their target genes to reduce their stability and/or inhibit translation, thus negatively regulating target gene expression. miRNAs are involved in a series of important pathophysiological processes, including cell proliferation, differentiation and apoptosis, as well as immune response and tumor formation $(8,9)$. It is estimated that miRNAs, which account for $1-3 \%$ of human genes, regulate the expression of $>30 \%$ of human genes (10). Recently, the role of miRNAs in the genesis and development of asthma has attracted increasing attention $(11,12)$. Furthermore, abnormal miRNA expression may be involved in asthmatic airway remodeling through regulating the proliferation and apoptosis of ASMCs (13-15). Let-7a, a member of the let-7 family, is one of the first identified miRNAs and one of the most abundant miRNAs in lung tissue (16). Let-7a regulates interleukin (IL)-13 secretion, while the latter has a vital role in the asthmatic inflammatory response and airway remodeling (17). Previous studies have indicated that let-7a expression is downregulated in bronchial epithelial 
cells and transbronchial lung biopsy tissues obtained during bronchoscopy from asthmatic patients $(18,19)$. Furthermore, silencing of let-7a was demonstrated to significantly alleviate airway inflammation and airway hyperreactivity in asthmatic mice $(20,21)$, suggesting that let-7a is associated with asthma. However, let-7a expression in bronchial SMCs from asthmatic patients as well as its regulatory actions have remained to be fully elucidated. In the present study, changes in let-7a expression levels in ASMCs from asthmatic vs. healthy patients were detected using reverse transcription-quantitative polymerase chain reaction (RT-qPCR), and the effect of let-7a on the proliferation and apoptosis of ASMCs was assessed; furthermore, potential target genes of let-7a were identified.

\section{Materials and methods}

Collection of patient samples and primary culture of human ASMCs. ASMCs from asthmatic and non-asthmatic subjects were derived from segmental bronchial biopsy specimens obtained through fiberoptic bronchoscopy at Shengli Oil Field Central Hospital (Dongying, China), including 15 asthmatic and 10 non-asthmatic subjects. None of the subjects had a history of smoking or of respiratory tract infection within the last 3 months. Asthmatic subjects conformed to the diagnostic criteria in the Guidelines for Prevention and Control of Bronchial Asthma in China (22). All subjects had provided written informed consent to participate in the study, and the present study was approved by the Ethics Committee of Shengli Oil Field Central Hospital (Dongying, China). Epithelium, connective tissue, blood vessels and cartilage were removed from the obtained bronchial biopsy specimens $(5 \times 5 \mathrm{~mm})$, followed by washing with the ice-cold PBS containing penicillin-streptomycin 3 times. The specimens were then placed in $5 \mathrm{ml}$ tissue culture flasks. Fetal bovine serum (FBS; $1 \mathrm{ml}$; 10\%; Gibco; Thermo Fisher Scientific, Inc., Waltham, MA, USA) was added, and the smooth muscle tissue block was cut into small pieces and repeatedly minced using iris scissors. The mixture was evenly applied onto the bottom surface of the culture flask with a sterile drinking straw. The culture flask was then inversely placed in a $5 \% \mathrm{CO}_{2}$ incubator at $37^{\circ} \mathrm{C}$. After $2 \mathrm{~h}$, when adherence of the cells was achieved, the flask was turned over and Dulbecco's modified Eagle's medium (Gibco; Thermo Fisher Scientific, Inc.) containing 10\% FBS was added from one side. The medium was replaced once every 3-4 days, and after 14 days of culture, hill and valley cell fusion was observed. Following detachment with $0.125 \%$ trypsin, the cells were passaged at a ratio of 1:2. Cells at passages 4-8 were used in the experiments. The ASMCs were identified by the typical 'hill and valley' growth pattern and immunocytochemical staining for $\alpha$-smooth muscle actin (23).

$R T-q P C R$. Total RNA of was extracted from the cells using TRIzol (Invitrogen; Thermo Fisher Scientific, Inc.), and the purity and content of the extracted RNA were determined using NanoDrop 2000 spectrophotometer (Thermo Fisher Scientific, Inc.) and RNA samples with a A260/A280 ratio between 1.8 and 2.0 were used to synthesize cDNA. Total RNA was reverse transcribed into cDNA using the High-Capacity cDNA Reverse Transcription kit (Thermo Fisher Scientific,
Inc.), according to the manufacturer's protocol. This reaction was performed at $25^{\circ} \mathrm{C}$ for $5 \mathrm{~min}, 50^{\circ} \mathrm{C}$ for $20 \mathrm{~min}$ then $75^{\circ} \mathrm{C}$ for 5 min. qPCR was performed with SYBR ${ }^{\circledR}$ Premix Ex Taq ${ }^{\mathrm{TM}}$ (Takara Bio Inc., Otsu, Japan; cat. no. DRR041A) according to the manufacturer's instructions, using an ABI 7500 real-time fluorescence qPCR machine (Applied Biosystems; Thermo Fisher Scientific, Inc.). U6 and $\beta$-actin were used as the internal reference genes for let-7a and signal transducer and activator of transcription (STAT3) detection, respectively. The reaction conditions for PCR were as follows: Initial denaturation at $95^{\circ} \mathrm{C}$ for $3 \mathrm{~min}$ and 40 cycles of $95^{\circ} \mathrm{C}$ for $15 \mathrm{sec}$ and $60^{\circ} \mathrm{C}$ for $30 \mathrm{sec}$. The primers used for the detection of let-7a and STAT3 were as follows: Let-7a forward, 5'-GCGCCTGAGGTAGTA GGTTG-3' and reverse, 5'-CAGTGCAGGGTCCGAGGT-3'; STAT3 forward, 5'-TGCTGGAGGAGAGAATCGT-3' and reverse, 5'-TAGTAGTGAACTGGACGCCG-3'; U6 forward, 5'-CTCGCTTCGGCAGCACA-3' and reverse, 5'-AACGCT TCACGAATTTGCGT-3'; $\beta$-actin forward, 5'-GGTCATCAC CATTGGCAA-3' and reverse, 5'-GAGTTGAAGGTAGTT TCGTGGA-3'. The primers were designed and synthesized by Shanghai Sangon Bioengineering Co., Ltd (Shanghai, China). The relative mRNA expression levels of let-7a and STAT3 were calculated using the $2^{-\Delta \Delta \mathrm{Cq}}$ method (24).

Cell transfection. ASMCs from asthmatic subjects in the logarithmic growth phase were inoculated into 6-well plates and divided into a let-7a mimics group and a negative control (NC)-mimics group. Let-7a mimics (5'-UGAGGUAGU AGgUUGUAUAGUU-3'; GenePharma, Shanghai, China) and NC-mimics (5'-UUCUCCGAACGUGUCACGUTT-3'; GenePharma) were transfected into the ASMCs of the corresponding groups using Lipofectamine ${ }^{\mathrm{TM}} 2000$ (Invitrogen; Thermo Fisher Scientific, Inc.) in strict accordance with the manufacturer's protocols. The transfection medium was replaced with normal medium at $6 \mathrm{~h}$ after transfection. The transfection efficiency was detected using RT-qPCR.

Cell Counting Kit (CCK)-8 assay. After 24 h of transfection, the cells were collected and seeded into the 96-well plates at a density of 3,000 cells/well. CCK-8 stain (10 $\mu \mathrm{l}$; Dojindo Molecular Technologies, Inc., Kumamoto, Japan) reaction liquid was added to designated wells every $24 \mathrm{~h}$ for $2 \mathrm{~h}$ of incubation at $37^{\circ} \mathrm{C}$, and the optical density (OD) value of each well was detected at the wavelength of $450 \mathrm{~nm}$ by ELX800 Universal Microplate Reader (BioTek Instruments, Inc., Winooski, VT, USA).

Flow cytometry. After $48 \mathrm{~h}$ of transfection, the cells were collected and washed with PBS twice, digested with EDTA-free trypsin and collected after centrifugation (111.8 x g, $5 \mathrm{~min}$, $\left.4^{\circ} \mathrm{C}\right)$ to prepare a single-cell suspension $\left(1 \times 10^{6}\right.$ cells $\left./ \mathrm{ml}\right)$ for each group individually. Of this cell suspension, $5 \mathrm{ml}$ was filled into a flow tube, followed by addition of $5 \mu \mathrm{l}$ Annexin V-fluorescein isothiocyanate (BD Biosciences) and $10 \mu 1$ propidium iodide (BD Biosciences) in succession. The mixtures were incubated for $15 \mathrm{~min}$ in the dark, followed by addition of $300 \mu \mathrm{l}$ binding buffer. The mixtures were then immediately subjected to flow cytometric evaluation (BD FACSCanto II; BD Biosciences, Franklin Lakes, NJ, USA) to determine the apoptotic rate. 
Caspase-3/7 activity assay. After $48 \mathrm{~h}$ of transfection, the cells were harvested and seeded onto the 96 -well plates. Substrates and buffer solution were defrosted at room temperature in strict accordance with the manufacturer's protocol for the Apo-ONE Homogeneous Caspase-3/7 Assay (Promega Corporation, Madison, WI, USA; cat. no. G7790). A total of $100 \mu 1$ substrate was mixed with $9,900 \mu 1$ buffer solution to prepare the Apo-ONE Caspase-3/7 reagent. Subsequently, $100 \mu \mathrm{l}$ of this reaction reagent was added to each well, followed by agitation using a shaker for $30 \mathrm{sec}$ and incubation for $2 \mathrm{~h}$ at room temperature in the dark. The fluorescence intensity of each well was then detected using a Tecan Infinite M200 pro plate reader (Tecan Trading AG, Maennedorf, Switzerland), from which the caspase-3/7 activity was determined.

Dual-luciferase reporter gene assay. Target genes of let-7a were predicted using the TargetScan miRNA target gene prediction bioinformatics website (http://www.targetscan.org). The bioinformatics prediction suggested that STAT3 may be a potential target gene for let-7a. The wild-type (WT) and mutant (MUT) reporter gene plasmids were synthesized by GeneCopoeia Co., Ltd. (Guangzhou, China). PCR amplification products of the 3'-untranslated region (3'-UTR) of STAT3 were connected to the XbalI enzyme digestion site of the pGL3 plasmid, so as to construct the wild-type pGL3-WT-STAT3 reporter gene plasmid (5'-UGACCUCGGAGUGCGCUA CCUCC-3'). Site-specific mutagenesis was performed on the binding site in the 3'UTR of STAT3 that is complementary to a sequence in let-7a, and the new plasmid obtained after mutagenesis was the pGL3-MUT-STAT3 reporter gene plasmid (5'-UGACCUCGGAGUGCGCAAGCACC-3'). 293 cells (ScienCell Research Laboratories, Inc., San Diego, CA, USA) were divided into 4 groups, namely the STAT3 WT plasmid control group (transfection with pGL3-WT-STAT3 reporter gene plasmid and NC-mimics), STAT3 WT plasmid experimental group (transfection with pGL3-WT-STAT3 reporter gene plasmid and let-7a mimics), STAT3 MUT plasmid control group (transfection with pGL3-MUT-STAT3 reporter gene plasmid and NC-mimics) and STAT3 MUT plasmid experimental group (transfection with pGL3-MUT-STAT3 reporter gene plasmid and let-7a mimics). Cells in each group were split after $24 \mathrm{~h}$ of transfection in accordance with the manufacturer's protocol for the dual-luciferase reporter gene system kit (Promega Corp.), and activities of firefly luciferase and Renilla luciferase in each group were detected using a GloMax 20/20 luminometer (Promega Corp.). Renilla luciferase gene was used as the internal reference to verify the transfection efficiency and calculate the relative luciferase activity as follows: Relative luciferase activity = firefly luciferase activity/Renilla luciferase activity.

Western blot analysis. Cells in each group were collected and washed with PBS for 3 times, followed by addition of radioimmunoprecipitation assay lysis buffer (Fermentas; Thermo Fisher Scientific, Inc.) and $1 \%$ protease inhibitor (cat. no. 78439; Pierce; Thermo Fisher Scientific, Inc.), and incubation for $20 \mathrm{~min}$ on ice. The mixtures were centrifuged $(111.8 \times \mathrm{g})$ at $4^{\circ} \mathrm{C}$ for $15 \mathrm{~min}$ and the supernatant was collected to determine the protein concentration using the bicinchoninic acid method. A total of $20 \mu \mathrm{g}$ protein per lane was separated

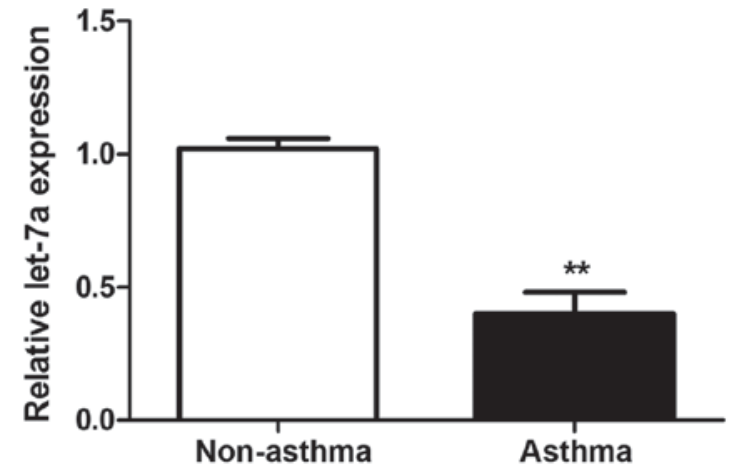

Figure 1. Let-7a expression levels in airway smooth muscle cells from asthmatic subjects were markedly downregulated compared with those from non-asthmatic subjects. ${ }^{* *} \mathrm{P}<0.01$ vs. non-asthma group.

by $10 \%$ SDS-PAGE, followed by transfer onto the polyvinylidene fluoride membranes (EMD Millipore, Billerica, MA, USA). Subsequent to blocking with $5 \%$ skimmed milk at room temperature for $6 \mathrm{~h}$, the membranes were incubated with primary antibodies against STAT3 (cat. no. sc-293151; 1:1,000 dilution) and GAPDH (cat. no. sc-32233; 1:1,000 dilution; Santa Cruz Biotechnology, Inc., Dallas, TX, USA) at $4^{\circ} \mathrm{C}$ overnight. The membrane was washed prior to the addition of horseradish peroxidase-conjugated secondary antibody (cat. no. sc-2354; 1:2,000 dilution; Santa Cruz Biotechnology, Inc.) and incubation at $37^{\circ} \mathrm{C}$ for $1 \mathrm{~h}$. The membrane was washed and protein bands were visualized using the enhanced chemiluminescence reagent (Western Blotting Detection kit; Applygen Technologies,Inc., Beijing, China) according the manufacturer's protocol. Subsequently, QuantityOne software (version 4.6; Bio-Rad Laboratories, Inc., Hercules, CA, USA) was used for densitometric analysis, and GAPDH was used as the internal reference to determine the relative expression of STAT3.

Statistical analysis. Data were analyzed by SPSS 19.0 software (IBM Corp., Armonk, NY, USA). Values are expressed as the mean \pm standard deviation and comparisons between groups were performed using the Student's t-test. All experiments were repeated at least 3 times. $\mathrm{P}<0.05$ was considered to indicate a statistically significant difference.

\section{Results}

Let-7a is downregulated in asthmatic ASMCs. The relative expression levels of let-7a in ASMCs from asthmatic and non-asthmatic subjects were detected using RT-qPCR. As presented in Fig. 1, the relative expression levels of let-7a in ASMCs from asthmatic subjects were significantly decreased compared with those in the ASMCs from non-asthmatic subjects $(\mathrm{P}<0.01)$.

Let-7a mimics inhibit the proliferation and promote the apoptosis of asthmatic ASMCs. ASMC proliferation has a vital role in airway remodeling (4). In order to determine the potential effect of let-7a on the proliferation of asthmatic ASMCs, asthmatic ASMCs were transfection with let-7a mimics, and the successful upregulation of let-7a was confirmed by RT-qPCR $(\mathrm{P}<0.01$; Fig. 2A). The results of the CCK-8 assay 
A

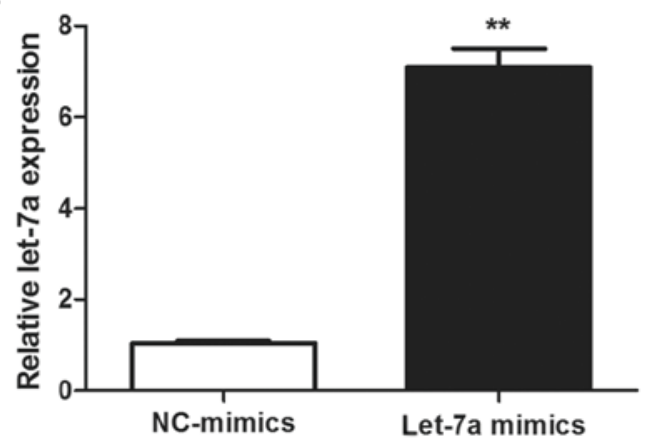

C

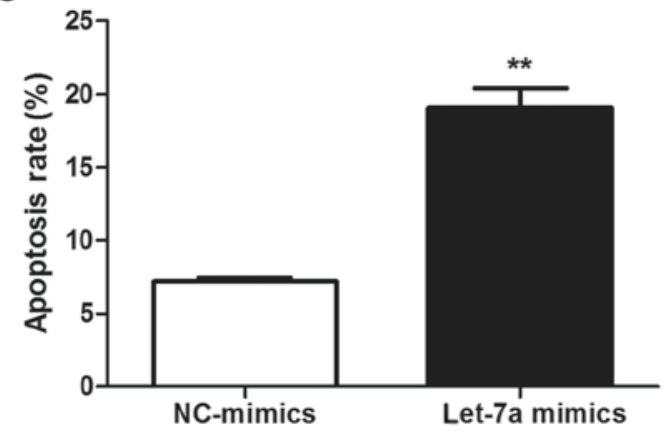

B

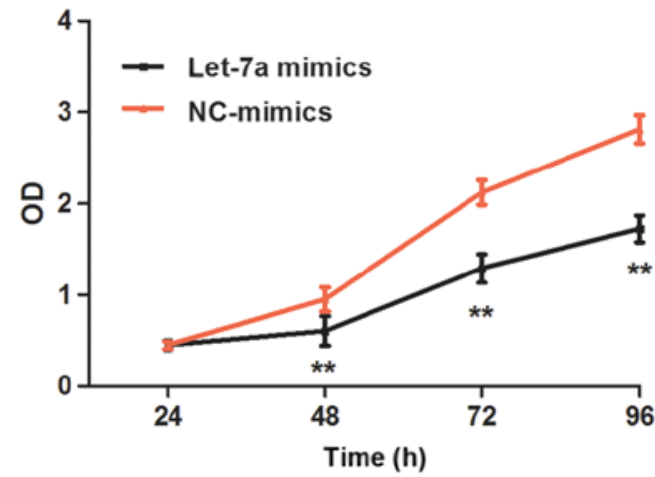

D

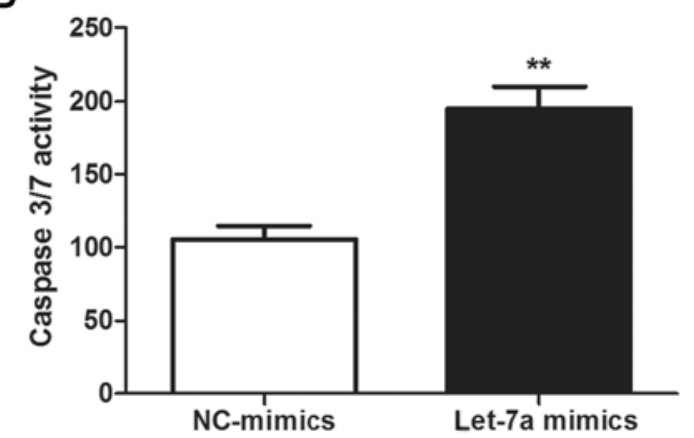

Figure 2. Upregulation of let-7a inhibits the proliferation and promotes apoptosis of asthmatic ASMCs. (A) Compared with the NC-mimics group, let-7a expression levels in the let-7a mimics group were markedly upregulated. (B) A Cell Counting Kit-8 assay indicated that let-7a mimics significantly suppressed the proliferation of asthmatic ASMCs. (C) Flow cytometric analysis indicated that let-7a mimics markedly promoted apoptosis of asthmatic ASMCs. (D) Caspase-3/7 activity analysis indicated that let-7a mimics notably promoted caspase-3/7 activity of asthmatic ASMCs. ${ }^{* *} \mathrm{P}<0.01 \mathrm{vs}$. NC mimics. OD, optical density; ASMCs, airway smooth muscle cells; NC, negative control.

A

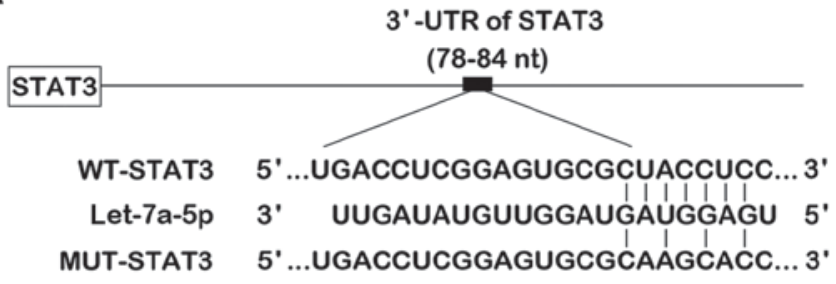

B

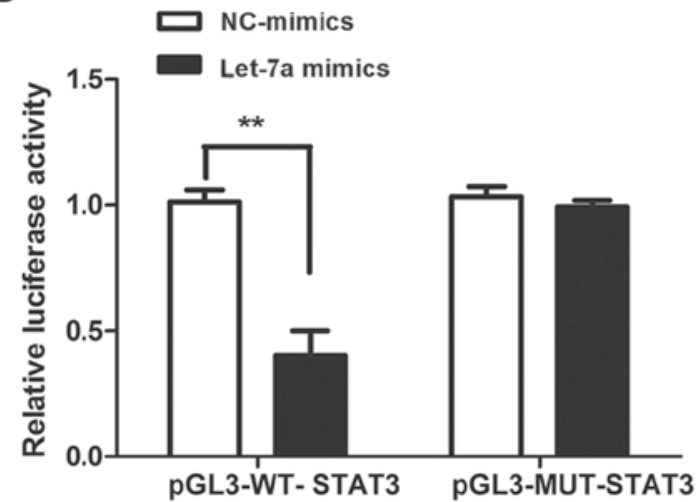

Figure 3. STAT3 was validated as a direct target gene of let-7a. (A) let-7a and STAT3 complementary binding sequences. (B) Let-7a mimics evidently suppressed the luciferase activity of wild-type pGL3-WT-STAT3, while NC mimics had no such effect. ${ }^{* *} \mathrm{P}<0.01$ vs. NC mimics. STAT3, signal transducer and activator of transcription; WT, wild-type; MUT, mutated; UTR, untranslated region.

indicated that let-7a mimics significantly suppressed the proliferation of asthmatic ASMCs $(\mathrm{P}<0.01$; Fig. 2B). Flow cytometric analysis indicated that let-7a mimics significantly enhanced the apoptosis of asthmatic ASMCs ( $\mathrm{P}<0.01$; Fig. 2C). Furthermore, let-7a mimics significantly increased the activity of caspase-3/7 in asthmatic ASMCs ( $\mathrm{P}<0.01$; Fig. 2D).

STAT3 is the direct regulatory target gene of let-7a. To determine the precise molecular biological mechanisms by which let-7a regulates the proliferation and apoptosis of asthmatic ASMCs, potential target genes of let-7a were analyzed and predicted using TargetScan. The analysis suggested that STAT3 is a potential target gene of let-7a due to the presence of theoretical complementary binding sites in the 3'-UTR of STAT3 mRNA with the seed sequence of let-7a (Fig. 3A). To further verify whether STAT3 is a direct target gene of let-7a, a dual-luciferase reporter gene assay was performed to confirm the effect of let-7a on the luciferase activity of the 3'-UTR of 
A

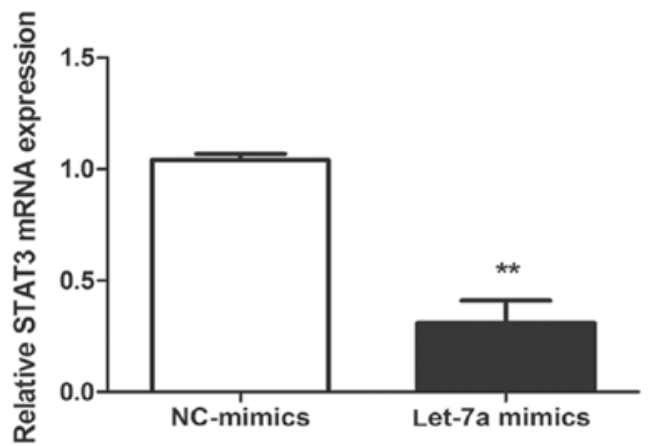

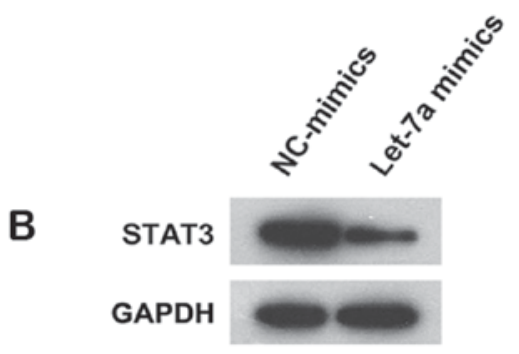

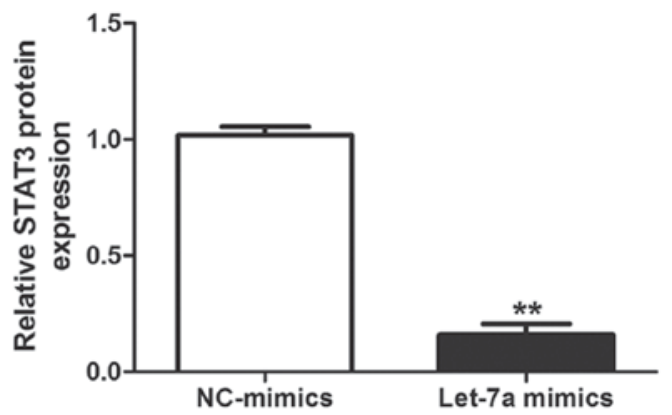

Figure 4. Let-7a mimics inhibited the expression of STAT3 mRNA and protein in asthmatic ASMCs. (A) Reverse transcription-quantitative polymerase chain reaction analysis demonstrated that let-7a mimics significantly reduced the expression levels of STAT3 mRNA in asthmatic ASMCs. (B) Western blot analysis demonstrated that upregulation of let-7a apparently reduced the expression levels of STAT3 protein in asthmatic ASMCs. ${ }^{* *} \mathrm{P}<0.01$ vs. NC mimics. STAT3, signal transducer and activator of transcription; ASMCs, airway smooth muscle cells.

STAT3 mRNA. As presented in Fig. 3B, let-7a mimics evidently suppressed the luciferase activity of the pGL3-WT-STAT3 reporter gene vector, while they had no obvious inhibitory effect on the luciferase activity of the pGL3-MUT-STAT3 reporter gene vector $(\mathrm{P}<0.01)$. This revealed that let-7a specifically binds with the 3'-UTR of the STAT3 mRNA, and that STAT3 is a direct target gene of let-7a.

Let-7a mimics inhibit the $m R N A$ and protein expression of STAT3 in asthmatic ASMCs. Finally, the mRNA and protein expression of STAT3 in asthmatic ASMCs transfected with let-7a mimics or NC-mimics was detected by RT-qPCR and western blot analysis, respectively. The expression levels of STAT3 mRNA and protein in the let-7a mimics group were significantly lower than those in the NC-mics group ( $\mathrm{P}<0.01$; Fig. 4A and $\mathrm{B})$, suggesting that upregulation of let-7a significantly inhibits the expression of STAT3 mRNA and protein in asthmatic ASMCs, further confirming that STAT3 is a target regulated by let-7a.

\section{Discussion}

Asthma is a common respiratory tract disease, which is characterized by airway remodeling and airway hyperreactivity. Airway remodeling refers to a series of structural changes in the airway wall under the persistent stimulation of mitogens, which are featured by epithelial damage, gland hyperplasia and hypertrophy, ASMC proliferation, ECM deposition and basilar membrane thickening. Of these, ASMC proliferation is the pathological foundation leading to structural changes in airway tissues (25), which not only aggravate airway stenosis but also increase airway hyperreactivity, thus having a crucial role in the genesis and development of airway remodeling (26). As a result, the prevention of ASMC proliferation to control the genesis of asthmatic airway remodeling has become a hotspot of current asthma research. In the last decade, an increasing number of studies have focused on ASMCs in asthma and have proposed to target them as a therapeutic strategy $(27,28)$.

Certain miRNAs have been verified to participate in the regulation of various biological processes, including cell proliferation, differentiation, apoptosis and tissue development through regulating target gene expression, and to have a key role in the genesis and development of multiple diseases $(29,30)$. Existing data regarding miRNAs and bronchial asthma suggest that miRNAs are involved in almost all pathophysiological processes of bronchial asthma, including allergic inflammation in the airways dominated by eosinophil infiltration, ASMC hyperreactivity and airway remodeling (11). In addition, patients with different severities of asthma may exhibit differences in their miRNA expression profile (31). Furthermore, multiple physiological functions of ASMCs, including contraction, proliferation and apoptosis, are also regulated by miRNAs. Hu et al (32) reported that overexpression of miR-10a markedly reduced the proliferation of human ASMCs, while inhibition of miR-10a promoted their proliferation. The underlying mechanisms included the specific inhibition of the phosphatidylinositide- 3 kinase catalytic subunit $\alpha$ by miR-10a, which blocked the AKT signaling pathway, as well as cyclins and cyclin-dependent kinases. Furthermore, another study identified that ras homolog family member A (RhoA) expression in ASMCs is negatively regulated by miR-133a, while IL-13 caused an upregulation of RhoA through reducing the miR-133a content in ASMCs. RhoA was identified as the key molecule to induce ASMC dysfunction, which may lead to excessive contraction, abnormal proliferation and apoptotic disorders of ASMCs (33). Therefore, correcting the aberrant expression of critical miRNAs in ASMC may potentially delay airway remodeling and the associated decline in lung function, which may be a promising approach for treating asthma. 
Let-7a is a member of the let-7a family, the aberrant expression of which has been identified in multiple diseases, including malignancies, Alzheimer's disease, asthma, allergic rhinitis and allergic dermatitis (34). Solberg et al (18) verified through miRNA microarrays combined with RT-qPCR that let-7a is markedly downregulated in bronchial epithelial cells of asthmatic patients. Rijavec et al (19) reported that let-7a was distinctly downregulated in transbronchial lung biopsy tissues obtained during bronchoscopy from patients with severe asthma compared with patients with mild asthma and non-asthmatic controls. Levänen et al (35) discovered that the levels of let-7a were obviously decreased in the broncho-alveolar lavage fluid of asthmatic patients. However, to the best of our knowledge, the expression of let-7a in asthmatic ASMCs has not been previously reported. The present study indicated that let-7a is notably downregulated in ASMCs from asthmatic subjects. Therefore, it may be speculated that aberrant let-7a expression is associated with the dysfunction of ASMCs in asthmatic patients. Johnson et al (36) suggested that Let-7a inhibited pathways controlling cell proliferation, and may also be the major regulator of cell proliferation. Furthermore, Cheng et al (37) discovered that upregulation of let-7a expression in bone mesenchymal stem cells suppressed the proliferation of pulmonary artery SMCs through the STAT3/bone morphogenetic protein receptor type 2 signaling pathway, thus delaying the progression of pulmonary hypertension. Furthermore, it has been verified that upregulation of let-7a expression markedly inhibits the proliferation of vascular SMCs (38); in addition, let-7a inhibits the proliferation of multiple types of tumor cell and promotes tumor cell apoptosis (39). In the present study, to determine whether aberrant let-7a expression in asthmatic ASMCs affects their proliferation and apoptosis, ASMCs from asthmatic patients were transfected with let-7a mimics to successfully upregulate let-7a expression. A CCK-8 assay indicated that let-7a mimics markedly suppressed the proliferation of asthmatic ASMCs. Furthermore, flow cytometric analysis and a caspase-3/7 activity assay suggested that let-7a mimics markedly enhanced the apoptosis and caspase-3/7 activity of asthmatic ASMCs, thereby verifying that let-7a affects the remodeling process of asthmatic airway tissues through suppressing the proliferation and promoting the apoptosis of asthmatic ASMCs.

The miRNA target prediction database TargetScan was then used to further explore the downstream targeted regulatory mechanisms by which let-7a regulates the function of asthmatic ASMCs. The bioinformatics prediction suggested that let-7a directly and specifically regulates STAT3, as theoretical complementary binding sites in the 3'-UTR of STAT3 mRNA with the seed sequence of let-7a were identified. Furthermore, let-7a was confirmed in previous studies to inhibit hepatoma cell proliferation through specifically regulating STAT3 gene expression (40), which also enhanced the sensitivity of hepatoma cells to cetuximab through specifically regulating the expression of the STAT3 gene (41). In the present study, the dual-luciferase assay indicated that let-7a specifically binds with the 3'-UTR of STAT3. In addition, RT-qPCR and western blot analysis indicated that upregulation of let-7a evidently reduces the mRNA and protein expression levels of STAT3 in asthmatic ASMCs, further suggesting that STAT3 is a target gene regulated by let-7a. STAT3 is a member of the STAT family, and is an important signal transcription factor in the Janus kinase/STAT signal transduction pathway. It possesses multiple important biological activities, and is involved in processes including cell proliferation, differentiation, survival, angiogenesis and cancer metastasis, through regulating the expression of various genes and cytokines (42). STAT3 has been verified as a key regulatory factor in airway remodeling. Allergic asthma and airway hyperresponsiveness were significantly relieved in asthmatic mice with STAT3 knockout (43). Furthermore, STAT3 inhibitor was reported to significantly inhibit airway remodeling and lung inflammation in asthmatic mice (44). STAT3 was demonstrated to promote ASMC proliferation through inducing the expression of chemokines and growth factors (45). Knockout of STAT3 significantly inhibited the proliferation of SMCs in human airway tissues (46). Platelet-derived growth factor was reported to induce the proliferation of human ASMCs through activation of STAT3 (47). Shi et al (48) also confirmed that upregulation of STAT3 expression significantly promoted the proliferation of human ASMCs.

In conclusion, the present study suggested that let-7a is downregulated in ASMCs of asthmatic patients. In addition, let-7a was indicated to inhibit the proliferation and promote the apoptosis of human ASMCs, which may be associated with the downregulation of STAT3 expression. Let-7a has a vital role during asthmatic airway remodeling.

\section{Acknowledgements}

Not applicable.

\section{Funding}

The present study was supported by grants of the Natural and Science Foundation of Shandong Province (grant no. ZR2014HL003). The funders had no role in the study design, data collection and analysis, decision to publish or preparation of the manuscript.

\section{Availability of data and materials}

The datasets generated and analyzed during the study are available from the corresponding author on reasonable request.

\section{Authors' contributions}

HL designed the experiments. YC,LQ,ZZ, GH and JZ performed the experiments. YC and HL analyzed the data. HL wrote the manuscript.

\section{Ethics approval and consent to participate}

All subjects had provided written informed consent to participate in the study, and the present study was approved by the Ethics Committee of Shengli Oil Field Central Hospital (Dongying, China).

\section{Patient consent for publication}

Written informed consent was obtained from all patients prior to publication. 


\section{Competing interests}

The authors declare that they have no competing interests

\section{References}

1. Bergmann KC: Bronchial asthma-many types, different therapies. Dtsch Med Wochenschr 141: 687-692, 2016 (In German).

2. Wang HY, Wong GW, Chen YZ, Ferguson AC, Greene JM, Ma Y, Zhong NS, Lai CK and Sears MR: Prevalence of asthma among Chinese adolescents living in Canada and in China. CMAJ 179: 1133-1142, 2008.

3. Miller M, Rosenthal P, Beppu A, Mueller JL, Hoffman HM, Tam AB, Doherty TA, McGeough MD, Pena CA, Suzukawa M, et al: ORMDL3 transgenic mice have increased airway remodeling and airway responsiveness characteristic of asthma. J Immunol 192: 3475-3487, 2014

4. Perry MM, Durham AL, Austin PJ, Adcock IM and Chung KF: BET bromodomains regulate transforming growth factor- $\beta$-induced proliferation and cytokine release in asthmatic airway smooth muscle. J Biol Chem 290: 9111-9121, 2015.

5. Bergeron C, Tulic MK and Hamid Q: Airway remodelling in asthma: From benchside to clinical practice. Can Respir J 17: e85-93, 2010

6. Balmasova IP, Sepiashvili RI, Sepiashvili IaR and Malova ES: Bronchial asthma pathogenesis and genetic prognosis development. Zh Mikrobiol Epidemiol Immunobiol 3: 60-67, 2014 (In Russian).

7. Hizawa N: Bronchial asthma: Progress in diagnosis and treatments. Topics: II. Pathogenesis and pathophysiology; 2. Genes associated with asthma and asthma-related phenotypes. Nihon Naika Gakkai Zasshi 102: 1365-1369, 2013 (In Japanese).

8. Filipowicz W, Bhattacharyya SN and Sonenberg N: Mechanisms of post-transcriptional regulation by microRNAs: Are the answers in sight? Nat Rev Genet 9: 102-114, 2008.

9. Bartel DP: MicroRNAs: Genomics, biogenesis, mechanism, and function. Cell 116: 281-297, 2004.

10. Lewis BP, Burge CB and Bartel DP: Conserved seed pairing, often flanked by adenosines, indicates that thousands of human genes are microRNA targets. Cell 120: 15-20, 2005.

11. Perry MM, Adcock IM and Chung KF: Role of microRNAs in allergic asthma: Present and future. Curr Opin Allergy Clin Immunol 15: 156-162, 2015.

12. Kai W, Qian XU and Qun WU: MicroRNAs and Asthma Regulation. Iran J Allergy Asthma Immunol 14: 120-125, 2015.

13. Chen M, Shi J, Zhang W, Huang L, Lin X, Lv Z, Zhang W, Liang R and Jiang S: MiR-23b controls TGF- $\beta 1$ induced airway smooth muscle cell proliferation via direct targeting of Smad3. Pulm Pharmacol Ther 42: 33-42, 2017.

14. Liu Y, Yang K, Shi H, Xu J, Zhang D, Wu Y, Zhou S and Sun X: MiR-21 modulates human airway smooth muscle cell proliferation and migration in asthma through regulation of PTEN expression. Exp Lung Res 41: 535-545, 2015.

15. Zhang H, Sun Z, Yu L and Sun J: MiR-139-5p inhibits proliferation and promoted apoptosis of human airway smooth muscle cells by downregulating the Brg1 gene. Respir Physiol Neurobiol 246: 9-16, 2017.

16. Takamizawa J, Konishi H, Yanagisawa K, Tomida S, Osada H, Endoh H, Harano T, Yatabe Y, Nagino M, Nimura Y, et al: Reduced expression of the let-7 microRNAs in human lung cancers in association with shortened postoperative survival. Cancer Res 64: 3753-3756, 2004.

17. Zhang FQ, Han XP, Zhang F, Ma X, Xiang D, Yang XM, Ou-Yang HF and Li Z: Therapeutic efficacy of a co-blockade of IL-13 and IL-25 on airway inflammation and remodeling in a mouse model of asthma. Int Immunopharmacol 46: 133-140, 2017.

18. Solberg OD, Ostrin EJ, Love MI, Peng JC, Bhakta NR, Hou L, Nguyen C, Solon M, Nguyen C, Barczak AJ, et al: Airway epithelial miRNA expression is altered in asthma. Am J Respir Crit Care Med 186: 965-974, 2012.

19. Rijavec M, Korošec P, Žavbi M, Kern I and Malovrh MM: Let-7a is differentially expressed in bronchial biopsies of patients with severe asthma. Sci Rep 4: 6103, 2014.

20. Polikepahad S, Knight JM, Naghavi AO, Oplt T, Creighton CJ, Shaw C, Benham AL, Kim J, Soibam B, Harris RA, et al: Proinflammatory role for let-7 microRNAS in experimental asthma. J Biol Chem 285: 30139-30149, 2010.
21. Kumar M, Ahmad T, Sharma A, Mabalirajan U, Kulshreshtha A, Agrawal A and Ghosh B: Let-7 microRNA-mediated regulation of IL-13 and allergic airway inflammation. J Allergy Clin Immunol 128: 1077-1085.e1-10, 2011.

22. Asthma Workgroup; Chinese Thoracic Society; Chinese Societ of General Practitioners: Chinese guideline for the prevention and management of bronchial asthma (Primary Health Care Version). J Thorac Dis 5: 667-677, 2013.

23. Chen M, Huang L, Zhang W, Shi J, Lin X, Lv Z, Zhang W, Liang R and Jiang S: MiR-23b controls TGF- $\beta 1$ induced airway smooth muscle cell proliferation via TGF $\beta R 2 / p-S m a d 3$ signals. Mol Immunol 70: 84-93, 2016.

24. Livak KJ and Schmittgen TD: Analysis of relative gene expression data using real-time quantitative PCR and the 2(-Delta Delta C(T)) method. Methods 25: 402-408, 2001.

25. Li LH, Lu B, Wu HK, Zhang H and Yao FF: Apigenin inhibits TGF- $\beta 1$-induced proliferation and migration of airway smooth muscle cells. Int J Clin Exp Pathol 8: 12557-12563, 2015.

26. Fahy JV, Corry DB and Boushey HA: Airway inflammation and remodeling in asthma. Curr Opin Pulm Med 6: 15-20, 2000.

27. Baroffio M, Crimi E and Brusasco V: Airway smooth muscle as a model for new investigative drugs in asthma. Ther Adv Respir Dis 2: 129-139, 2008.

28. Dowell ML, Lavoie TL, Solway J and Krishnan R: Airway smooth muscle: A potential target for asthma therapy. Curr Opin Pulm Med 20: 66-72, 2014.

29. Brase JC, Wuttig D, Kuner R and Sültmann H: Serum microRNAs as non-invasive biomarkers for cancer. Mol Cancer 9: 306, 2010.

30. Lovat F, Valeri N and Croce CM: MicroRNAs in the pathogenesis of cancer. Semin Oncol 38: 724-733, 2011.

31. Tsitsiou E, Williams AE, Moschos SA, Patel K, Rossios C, Jiang X, Adams OD, Macedo P, Booton R, Gibeon D, et al: Transcriptome analysis shows activation of circulating CD8+ T cells in patients with severe asthma. J Allergy Clin Immunol 129: 95-103, 2012.

32. Hu R, Pan W, Fedulov AV, Jester W, Jones MR, Weiss ST, Panettieri RA Jr, Tantisira K and Lu Q: MicroRNA-10a controls airway smooth muscle cell proliferation via direct targeting of the PI3 kinase pathway. FASEB J 28: 2347-2357, 2014.

33. Chiba Y, Tanabe M, Goto K, Sakai H and Misawa M: Down-regulation of miR-133a contributes to up-regulation of Rhoa in bronchial smooth muscle cells. Am J Respir Crit Care Med 180: 713-719, 2009.

34. Russo F, Di Bella S, Nigita G, Macca V, Laganà A, Giugno R, Pulvirenti A and Ferro A: miRandola: Extracellular circulating microRNAs database. PLoS One 7: e47786, 2012.

35. Levänen B, Bhakta NR, Torregrosa Paredes P, Barbeau R, Hiltbrunner S, Pollack JL, Sköld CM, Svartengren M, Grunewald J, Gabrielsson S, et al: Altered microRNA profiles in bronchoalveolar lavage fluid exosomes in asthmatic patients. J Allergy Clin Immunol 131: 894-903, 2013.

36. Johnson CD, Esquela-Kerscher A, Stefani G, Byrom M, Kelnar K, Ovcharenko D, Wilson M, Wang X, Shelton J, Shingara J, et al: The let-7 microRNA represses cell proliferation pathways in human cells. Cancer Res 67: 7713-7722, 2007.

37. Cheng G, Wang X, Li Y and He L: Let-7a-transfected mesenchymal stem cells ameliorate monocrotaline-induced pulmonary hypertension by suppressing pulmonary artery smooth muscle cell growth through STAT3-BMPR2 signaling. Stem Cell Res Ther 8: 34, 2017.

38. Cao H, Hu X, Zhang Q, Wang J, Li J, Liu B, Shao Y, Li X, Zhang J and Xin S: Upregulation of let-7a inhibits vascular smooth muscle cell proliferation in vitro and in vein graft intimal hyperplasia in rats. J Surg Res 192: 223-233, 2014.

39. Lee YS and Dutta A: The tumor suppressor microRNA let-7 represses the HMGA2 oncogene. Genes Dev 21: 1025-1030, 2007.

40. Wang Y, Lu Y, Toh ST, Sung WK, Tan P, Chow P, Chung AY, Jooi LL and Lee CG: Lethal-7 is down-regulated by the hepatitis $B$ virus $x$ protein and targets signal transducer and activator of transcription 3. J Hepatol 53: 57-66, 2010.

41. Xue F, Liu Y, Zhang H, Wen Y, Yan L, Tang Q, Xiao E and Zhang D: Let-7a enhances the sensitivity of hepatocellular carcinoma cells to cetuximab by regulating STAT3 expression. Onco Targets Ther 9: 7253-7261, 2016.

42. Aggarwal BB, Kunnumakkara AB, Harikumar KB, Gupta SR, Tharakan ST, Koca C, Dey S and Sung B: Signal transducer and activator of transcription-3, inflammation, and cancer: How intimate is the relationship?. Ann N Y Acad Sci 1171: 59-76, 2009.

43. Simeone-Penney MC, Severgnini M, Tu P, Homer RJ, Mariani TJ, Cohn L and Simon AR: Airway epithelial STAT3 is required for allergic inflammation in a murine model of asthma. J Immunol 178: 6191-6199, 2007 
44. Gavino AC, Nahmod K, Bharadwaj U, Makedonas G and Tweardy DJ: STAT3 inhibition prevents lung inflammation, remodeling, and accumulation of Th2 and Th17 cells in a murine asthma model. Allergy 71: 1684-1692, 2016.

45. Simeone-Penney MC, Severgnini M, Rozo L, Takahashi S, Cochran BH and Simon AR: PDGF-induced human airway smooth muscle cell proliferation requires STAT3 and the small GTPase Rac1. Am J Physiol Lung Cell Mol Physiol 294: L698-L704, 2008.

46. Redhu NS, Shan L, Movassagh H and Gounni AS: Thymic stromal lymphopoietin induces migration in human airway smooth muscle cells. Sci Rep 3: 2301, 2013.
47. Simon AR, Takahashi S, Severgnini M, Fanburg BL and Cochran BH: Role of the JAK-STAT pathway in PDGF-stimulated proliferation of human airway smooth muscle cells. Am J Physiol Lung Cell Mol Physiol 282: L1296-L1304, 2002.

48. Shi S, Jin L, Zhang S,Li H,Zhang B and Sun M: MicroRNA-590-5p represses proliferation of human fetal airway smooth muscle cells by targeting signal transducer and activator of transcription 3 . Arch Med Sci 14: 1093-1101, 2018.

(i) (9) This work is licensed under a Creative Commons cc) Attribution-NonCommercial-NoDerivatives 4.0 International (CC BY-NC-ND 4.0) License. 